\title{
Un recorrido por las propiedades microestructurales de la aleación Al-Fe-Cr
}

\author{
An overview for the microstructural properties of the Al-Fe-Cr alloy

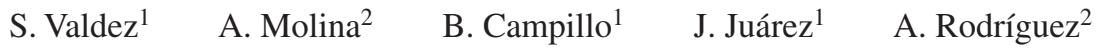 \\ Recibido 5 de septiembre de 2011, aceptado 5 de abril de 2013 \\ Received: September 5, $2011 \quad$ Accepted: April 5, 2013
}

\begin{abstract}
RESUMEN
La aleación Al-Fe-Cr ha sido solidificada rápidamente por la técnica de melt-spinning. La estructura de los listones ha sido estudiada por SEM, TEM y Difracción de rayos-X. Los listones fueron recocidos a $500{ }^{\circ} \mathrm{C}$ durante $48 \mathrm{hrs}$. para estudiar la influencia del tratamiento térmico sobre la microestructura. $\mathrm{La}$ microestructura de los listones de la aleación ternaria $\mathrm{Al}-\mathrm{Fe}-\mathrm{Cr}$ sufrió un considerable refinamiento en comparación con la misma aleación producida por el método convencional de fusión y colado. El tamaño de grano de los listones decrece tanto como la velocidad de enfriamiento incrementa. Una estructura ordenada del tipo B2 fue obtenida para las aleaciones ternarias solidificadas rápidamente y recocidas en todas las velocidades de enfriamiento.
\end{abstract}

Palabras clave: Solidificación rápida, enfriamiento ultrarrápido, aluminuros de hierro, microestructura, técnica de Melt-spinning.

\section{ABSTRACT}

Al-Fe-Cr alloy was rapidly solidified by means of melt spinning technique. Alloy structure has been studied by SEM, TEM and X-Ray diffraction. The ribbons were annealing at $500{ }^{\circ} \mathrm{C}$ during 48 hours, in order to study the influence of thermal treatment onto the Al-Fe-Cr microstructure. The melt-spun Al$\mathrm{Fe}$-Cr microstructure underwent a considerable refinement as compared with same composition alloy produced by conventional methods of melting and casting. In melt-spun ribbons the grain size decreases as the cooling increases. An ordered B2 type structure was obtained in all ternary rapidly solidified and annealed alloys at all cooling rates schedules.

Keywords: Rapid solidification, ultrafast cooling, iron aluminides, microstructure, melt-spinning technique.

\section{INTRODUCCIÓN}

Los compuestos intermetálicos ordenados son materiales candidatos para aplicaciones estructurales a temperaturas elevadas [1]. Sin embargo, a temperatura ambiente tienen aplicaciones limitadas debido a su baja ductilidad y baja resistencia al impacto [2]. A través de un preciso control en la composición y la microestructura se pueden mejorar considerablemente sus propiedades mecánicas. Las rutas que permiten este control son la solidificación rápida, recocido, tratamiento termomecánico y aleado convencional [3]. Mediante el micro y macroaleado con Ti, V, Nb, $\mathrm{Ta}, \mathrm{Cr}, \mathrm{Mo}, \mathrm{W}, \mathrm{Si}$ y Ni los elementos que reducen la fragilización mejoran la ductilidad e inducen pasividad [4]. Otro método de gran utilidad es el proceso de solidificación rápida mediante melt-spinning, el cual promete ser un medio de estudio para correlacionar la velocidad de enfriamiento con la microestructura y las propiedades mecánicas de compuestos intermetálicos.

\footnotetext{
1 Instituto de Ciencias Físicas. Universidad Nacional Autónoma de México. Av. Universidad S/N. Cuernavaca. C.P. 62210. Morelos, México. E-mail: svaldez@ fis.unam.mx; campillo@unam.mx; juarez@unam.mx

2 Centro de Investigación en Ingeniería y Ciencias Aplicadas. Universidad Autónoma del Estado de Morelos. Av. Universidad 1001. Cuernavaca. C.P. 82210. Morelos, México.E-mail: amolina@uaem.mx; arodriguez@uaem.mx
} 
La ductilidad reportada para la aleación Al-40Fe$5 \mathrm{Cr}$ obtenida mediante extrusión y recocido ha sido correlacionada también con el tipo de procesamiento y la microestructura [5]. Para este sistema se han reportado alargamientos de un $2 \%$ y $2,2 \%$ para tamaños de grano de cinco micras. Sin embargo, reportan la presencia de partículas de $\mathrm{Cr}_{2} \mathrm{Al}$ originando un incremento en la resistencia a la fluencia con una reducción simultánea de la ductilidad. Para nuestro sistema se ha considerado evitar la precipitación de partículas de $\mathrm{Cr}_{2} \mathrm{Al}$, que limitan la capacidad de deformación. Para ello, se considera el procesamiento de solidificación rápida, con la finalidad adicional de mejorar la extensión de la solubilidad del soluto en la matriz férrica.

El propósito de este trabajo es dar a conocer el efecto de la solidificación rápida sobre la aleación de $\mathrm{AlFe}$ macroaleado con cromo $(\mathrm{Cr})$, así como la influencia que el cromo ejerce sobre la microestructura; en particular, se considera el parámetro de red, tamaño de grano y la solubilidad del soluto para condiciones de colado convencional, solidificación rápida y recocido.

\section{PROCEDIMIENTO EXPERIMENTAL}

\section{Síntesis de la aleación AlFeCr}

Los lingotes de Al-60Fe-5Cr bajo condición de colada se obtuvieron mediante un horno de inducción al vacío y con piezas de hierro, aluminio y cromo de alta pureza $(99,9 \%)$. La aleación fundida fue vaciada en un molde de cobre con forma rectangular. Pequeñas piezas cúbicas de $1 \mathrm{~cm}^{3}$ fueron cortadas con un disco de diamante para ser utilizadas en la fabricación de los listones mediante la técnica de melt-spinning aplicando diferentes velocidades tangenciales.

\section{Preparación de los listones}

Las piezas cúbicas fueron refundidas bajo una atmósfera de argón dentro de un tubo de cuarzo, el cual tiene un orificio de salida de $1 \mathrm{~mm}$ de diámetro. Con la aleación fundida en el tubo de cuarzo se inyecta gas argón a 34,47 KPa con la finalidad de dejar caer el líquido sobre una superficie de cobre, que nos permitió obtener velocidades de enfriamiento de $0,715 \times 10^{6} \mathrm{~K} / \mathrm{s}$ a $1,38 \times 10^{6} \mathrm{~K} / \mathrm{s}$. Las cintas o listones obtenidos bajo estas condiciones presentan un espesor que varía de 20 a 90 micras y de 2 a 3 $\mathrm{mm}$ de ancho. Estas cintas fueron posteriormente recocidas a $500{ }^{\circ} \mathrm{C}$ durante $48 \mathrm{~h}$ para inducir la eliminación de sitios vacantes.

\section{Preparación metalográfica}

La superficie tanto de las muestras obtenidas por solidificación rápida como por los listones sometidos a recocido fue preparada metalográficamente en la sección de área transversal en contacto con la superficie de cobre. La preparación metalográfica se llevó a cabo con la técnica estándar de desbaste con papel lija de carburo de silicio de \#240 a \#1500. Terminado el proceso de desbaste y limpieza de las muestras, se procedió con la etapa del pulido, llevado a cabo mecánicamente con alúmina en polvo de $1 \mu \mathrm{m}$. La muestra en condición de colada fue atacada con una mezcla de $50 \% \mathrm{CH}_{3} \mathrm{COOH}+$ $33 \% \mathrm{HNO}_{3}+17 \% \mathrm{HCl}$, mientras que los listones fueron atacados con reactivo Keller. Mediante este procedimiento es posible un revelado del grano que permita determinar su tamaño haciendo uso de la norma ASTM 11-2.

\section{Caracterización Microestructural}

Para observar el tamaño de grano y la morfología de la aleación se utilizó un microscopio óptico metalográfico y un microscopio electrónico de barrido marca Leica Cambridge modelo STEREOSCAN-440 equipado con espectroscopia de dispersión de energía (OXFORD Isis 300) empleado para realizar análisis químico puntual. Los detalles de la microestructura fina fueron examinados a través de un microscopio de transmisión JEOL-2010. Para ello, se prepararon láminas de 50 micras de espesor, perforadas por electropulido usando una solución de $\mathrm{HClO}_{4}$ en etanol al $10 \%$ en volumen.

Para determinar la estructura cristalina e identificar cualitativamente las fases, se empleó un difractómetro de rayos X Siemens 5000 con radiación de cobre.

\section{RESULTADOS Y DISCUSIÓN}

\section{Caracterización Microestructural}

Los análisis metalográficos se realizaron para los listones a velocidades tangenciales de 12,16 y $20 \mathrm{~m} / \mathrm{s}$.

La Figura 1 muestra la micrografía óptica de los listones bajo las diferentes condiciones. En todos los listones obtenidos, los de solidificación rápida y los recocidos poseen una estructura cúbica de tipo B2. Aun con la adición de cromo al sistema Al-Fe, 


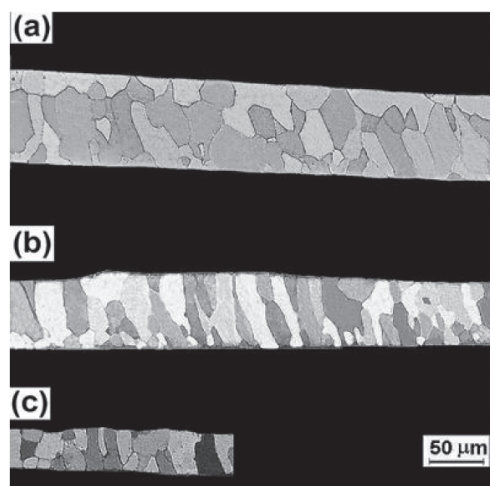

Figura 1. Microestructura de los listones de Al$\mathrm{Fe}-\mathrm{Cr}$ a velocidades tangenciales de (a) 12 , (b) 16 y (c) $20 \mathrm{~m} / \mathrm{s}$.

no se identifica la precipitación de partículas ricas en cromo, así como tampoco segregaciones macro o microestructurales de los aleantes hierro, cromo. Esto fue también confirmado mediante análisis por difracción de rayos X (Figura 3). Por otro lado, los resultados de los microanálisis lineales (EDSSEM) revelan una distribución uniforme de los elementos químicos $\mathrm{Al}$, Cr y Fe tanto en los listones solidificados rápidamente como en los recocidos. De la Figura 1(a) y 1(b) se observa que los listones obtenidos a velocidad tangencial de $12 \mathrm{~m} / \mathrm{s}$ presentan morfología de grano equiaxial externo, columnar. Mientras que para los listones obtenidos a $16 \mathrm{~m} / \mathrm{s}$ predomina la morfología de grano columnar. Por el contrario, a los listones fabricados a $20 \mathrm{~m} / \mathrm{s}$ la morfología principal es la equiaxial.

El tamaño de grano de los listones sufrió una drástica reducción respecto de la velocidad de enfriamiento, específicamente entre las muestras obtenidas por colada convencional respecto aquellas solidificadas rápidamente. El tamaño de los granos equiaxiales en los listones exhibe una reducción desde $24,7 \mu \mathrm{m}$ a 6,61 $\mu \mathrm{m}$ cuando la velocidad tangencial varía de 12 a $20 \mathrm{~ms}^{-1}$. Mientras que el ancho en los granos se reduce de 30,4 a 6,4 $\mu \mathrm{m}$ para los mismos valores de velocidad. Respecto de los listones después del recocido se observa también una disminución en el tamaño de grano. Por lo que se considera que este comportamiento es análogo a un proceso de recristalización después de la deformación. La disminución en el tamaño de grano se explica debido a que durante la solidificación rápida una gran cantidad de defectos podrían haberse originado en los listones a partir de los cuales se formaron dislocaciones después del recocido.

La Figura 2 exhibe la relación que existe entre el espesor de los listones como una función de la velocidad de enfriamiento y la relación entre la velocidad tangencial y la velocidad de enfriamiento. En la Figura 2 se observa que el espesor de los listones disminuye a medida que la velocidad de enfriamiento incrementa. Además, se presenta la relación directa entre velocidad de enfriamiento y velocidad tangencial, toda vez que la velocidad tangencial es mayor, la velocidad de enfriamiento incrementa también.

La relación entre espesor del listón $(\mathrm{t}, \mu \mathrm{m})$ y velocidad de giro de la rueda o velocidad tangencial $(\mathrm{V}, \mathrm{m} / \mathrm{s})$ puede estar relacionada con la siguiente expresión matemática: $\mathrm{t} \alpha \mathrm{V}^{-0,6}$. Esta formulación fue expresada como t $\alpha \mathrm{V}^{-0,8}[6]$ para el caso de vidrios metálicos solidificados rápidamente. De manera similar, se ha estudiado también la dependencia del espesor del listón (t) con la velocidad tangencial (V) en aleaciones cristalinas del tipo $\mathrm{Fe}-\mathrm{Cr}-\mathrm{Ni}$ [7] para la cual se halló la relación t $\alpha \mathrm{V}^{-0,6}$. En el caso de listones de Fe24Cr14Ni solidificados rápidamente el espesor está expresado como $\mathrm{t}=390 \mathrm{~V}^{-0,61}$ mientras que para una aleación de Fe15Cr10Ni y Fe13Cr11Ni se ha reportado una relación de $t=270 \mathrm{~V}^{-0,59}$. Sin embargo, la dependencia del espesor del listón con la velocidad tangencial varía ligeramente con las condiciones de fabricación y el sistema de aleación.

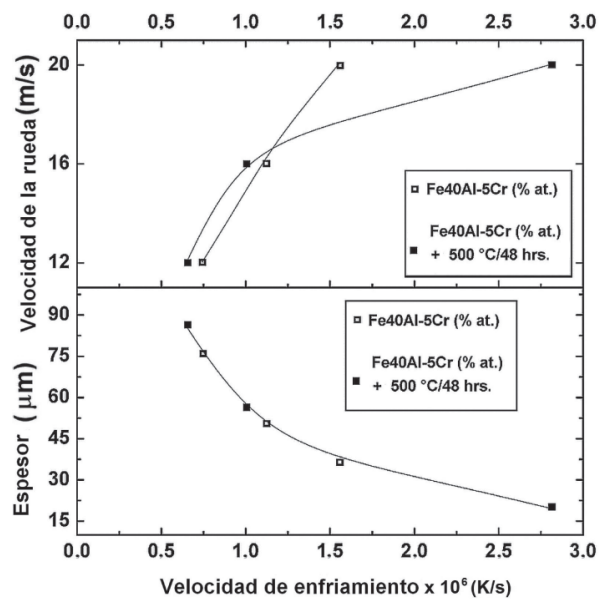

Figura 2. Relación entre el espesor de los listones y la velocidad de giro de la rueda respecto de la velocidad de enfriamiento. 


\section{Difracción de rayos $\mathrm{X}$}

La Figura 3 muestra los patrones de difracción de los listones en la condición de fusión y recocido. A partir de los difractogramas puede determinarse que el cromo no modifica la estructura ordenada tipo B2 independientemente de la velocidad tangencial o de la velocidad de enfriamiento. Los picos adicionales sugieren fases secundarias dado que no pertenecen al cromo y que no han sido aún identificadas, por lo que se intuye que el cromo forma parte de la solución sólida quedándose atrapado en la matriz de Al-Fe, lo que fue corroborado mediante los microanálisis de EDS-SEM. Los listones de Al-Fe-Cr exhiben los picos de difracción con mayor intensidad en (110) y (211).

Los parámetros de red de los listones con recocido presentan un ligero decremento en su valor, en comparación con los listones sin recocido, los valores de estos parámetros de red están expresados en la Tabla 1. Cabe señalar que se le atribuye al cromo una disminución en la concentración de vacancias, tal como ha sido revelado para el sistema $\mathrm{Al} 40 \mathrm{FeCr}$ reportado previamente [5]. Por lo tanto, se supone que esta disminución en el contenido de sitios vacantes origina el aumento del parámetro de red. En este caso, la reducción del parámetro de red después del recocido puede deberse a una combinación entre reducción de sitios vacantes y el mecanismo de reordenamiento atómico inducido por la adición de cromo.

La Figura 4 (a) esquematiza una imagen obtenida del microscopio electrónico de transmisión, en

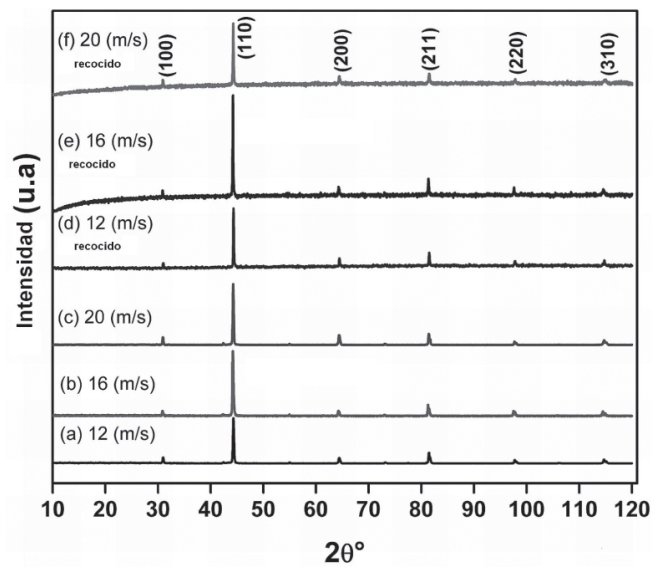

Figura 3. Perfiles de difracción de rayos X para los listones con y sin recocido.
Tabla 1. Parámetros microestructura de los listones ternarios Al-Fe-Cr.

\begin{tabular}{|c|c|c|}
\hline $\begin{array}{c}\text { Velocidad } \\
\text { Tangencial } \\
\text { V }\left(\mathbf{m s}^{-1}\right)\end{array}$ & $\begin{array}{c}\text { Tamaño } \\
\text { de } \\
\text { grano } \\
(\mu \mathrm{m})\end{array}$ & $\begin{array}{c}\text { Parámetro de } \\
\text { red } \\
\text { (a) } \AA\end{array}$ \\
\hline \multicolumn{3}{|c|}{ Listones sin recocido } \\
\hline 12 & 30,42 & 2,8898 \\
\hline 16 & 22,58 & 2,8963 \\
\hline 20 & 6,48 & 2,8933 \\
\hline \multicolumn{3}{|c|}{ Listones recocidos a $\left(500^{\circ} \mathrm{C} / 48 \mathrm{~h}\right)$} \\
\hline 12 & 21,59 & 2,8874 \\
\hline 16 & 13,27 & 2,8881 \\
\hline 20 & 6,39 & 2,8900 \\
\hline
\end{tabular}

donde se ejemplifica la unión de tres granos, y en su interior nanoporos originados probablemente por la agrupación de sitios vacantes. La morfología de los nanoporos es similar a la reportada en listones de NiAl y CoAl [8]. La formación de los nanoporos provenientes de sitios vacantes tiene su origen durante la solidificación rápida. Por otro lado, no se observan precipitados en límites de grano o matriz. A partir de la Figura 4(a), podemos también observar dislocaciones lineales. Estas dislocaciones pueden haberse formado debido a las temperaturas elevadas durante la fusión y atrapadas durante el rápido enfriamiento del proceso de solidificación rápida. Lo anterior sugiere que el cromo no interfiere significativamente en la formación de cúmulos de sitios vacantes, reiterando la formación a través del proceso de solidificación rápida. De la Figura 4(b) se puede observar el patrón de difracción con un eje de zona (111), el cual corresponde al área con nanoporos de la Figura 4(a). Por otro lado, la Figura 4(c) esquematiza una sección de la matriz Al-Fe.

\section{CONCLUSIONES}

En relación con los efectos por la adición de cromo sobre la microestructura, se han obtenido las siguientes conclusiones:

El análisis por TEM reveló que el cromo no interfiere significativamente en la generación de cúmulos de espacios vacantes, los que han sido atribuidos al enfriamiento del sistema $\mathrm{Al}-\mathrm{Fe}-\mathrm{Cr}$. 


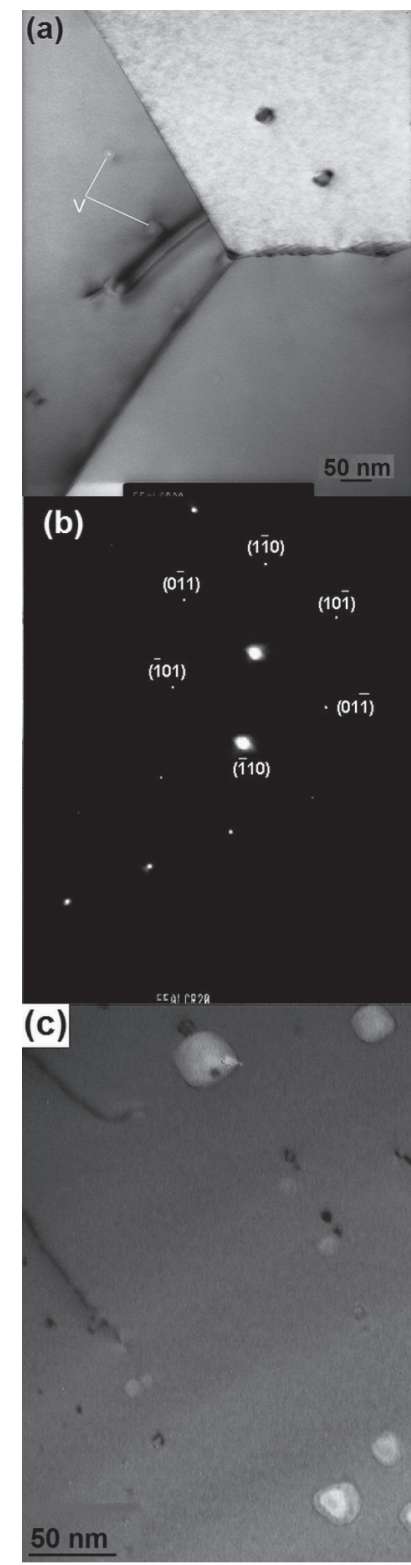

Figura 4. (a) y (c) Fotomicrografía obtenida por TEM correspondiente al listón de Al$\mathrm{Fe}-\mathrm{Cr}$ generado a $20 \mathrm{~m} / \mathrm{s}$, (b) patrón de difracción del grano en (a).

La adición de cromo no propicia la precipitación de partículas en matriz o límite de grano.

\section{AGRADECIMIENTOS}

Trabajo apoyado financieramente por los proyectos CONACYT-México No 167583 y DGAPA-PAPIIT-IT101112-3.

\section{REFERENCIAS}

[1] J.H. Westbrook and R.L. Fleischer. "Intermetallic compounds principles". J. Wiley and Sons Ltd. New York, USA. 1994.

[2] N.S. Stoloff. "Iron Aluminides: Present Status and Future Prospects". Mat. Sci. and Eng. Vol. A258, pp. 1-14. 1998.

[3] N.S. Stoloff and V.K. Sikka. "Physical Metallurgy and Processing of Intermetallic Compounds". Chapman \& Hall, pp. 56-89. 1996.

[4] H. Jones. "Rapid Solidification of Metals and Alloys". The Institute of Metallurgist. Monograph No 8. 1982.

[5] O. Klein and I. Baker. "Effect of chromium on the environmental sensitivity of $\mathrm{FeAl}$ at room temperature". Scrip. Met. et Mat. Vol. 27, pp. 1823-1828. 1992.

[6] E. J. Martínez D., M.A. Cisneros G., S. Valtierra and J. Lacaze. "Effect of Strontium and Cooling Rate Upon Eutectic Temperatures of A319 Aluminum Alloy". Scripta Materialia. Vol. 52, Issue 6, pp. 439-443. March 2005.

[7] L. Backerud, G. Chai and J. Tamminen. "Solidification Characteristics of Aluminum Alloys". AFS/SKANALUMINIUM. Vol. 2, pp. 71-229. 1990.

[8] R. Nakamura, K. Yoshimi and S. Tsurekawa. "Supersaturated vacancies and vacancy complexes in rapidly solidified B2 aluminide ribbons". Proccedings of the 12th International Conference on Rapidly Quenched \& Metastable Materials. Materials Science and Engineering: A. Elsevier. Vol. 449-451, pp. 1036-1040. 25 March 2007. 